\title{
Consistent Surface Color for Texturing Large Objects in Outdoor Scenes
}

\author{
Rei Kawakami and Katsushi Ikeuchi \\ The Universtity of Tokyo \\ \{rei,ki\}@ cvl.iis.u-tokyo.ac.jp
}

\author{
Robby T. Tan \\ NICTA -Australian National University \\ robby.tan@anu.edu.au
}

\begin{abstract}
Color appearance of an object is significantly influenced by the color of the illumination. When the illumination color changes, the color appearance of the object will change accordingly, causing its appearance to be inconsistent. To arrive at color constancy, we have developed a physics-based method of estimating and removing the illumination color. In this paper, we focus on the use of this method to deal with outdoor scenes, since very few physics-based methods have successfully handled outdoor color constancy. Our method is principally based on shadowed and non-shadowed regions. Previously researchers have discovered that shadowed regions are illuminated by sky light, while non-shadowed regions are illuminated by a combination of sky light and sunlight. Based on this difference of illumination, we estimate the illumination colors (both the sunlight and the sky light) and then remove them. To reliably estimate the illumination colors in outdoor scenes, we include the analysis of noise, since the presence of noise is inevitable in natural images. As a result, compared to existing methods, the proposed method is more effective and robust in handling outdoor scenes. In addition, the proposed method requires only a single input image, making it useful for many applications of computer vision.
\end{abstract}

\section{Introduction}

Reflected light from an object is the product of surface spectral reflectance and illumination spectral power distribution. Consequently, illumination color significantly determines the object's color appearance. When the illumination color changes, the object color appearance changes accordingly. This leads to many problems in algorithms of computer vision. For example, Figure 1 shows that the illumination change in creating a realistic model causes the color appearance of an object to be inconsistent. Recovering the surface's actual color requires a method of color constancy that discounts the inconsistencies caused by variations in illumination.

Color constancy is one important aspect of the field of computer vision. Many algorithms in this field, such as color-based object recognition, image retrieval, reflection component separation, and real object rendering, require recovery of the actual color of objects. Many methods have been proposed for this recovery $[3,9,18,5,7,11$, $15,16,10,12,17]$. Based on their input, we can categorize these methods into dichromatic-based methods and diffuse-based methods [17]. Dichromatic-based methods $[5,7,11,15,16,10,17]$ require the presence of highlighting, while diffuse-based methods $[3,9,18]$ require bodyonly reflection.

Most diffuse-based methods use a single input image of objects lit by a uniformly colored surface. Usually these methods require strong constraints in surface colors domain, such as a prior surface color database, and cannot accurately estimate images with few surface colors [18]. A few researchers alternatively introduce color constancy methods based on varying or changing illumination color $[4,8,2]$. They have found that, despite creating the problem of color constancy, the change of illuminations could be a crucial constraint to solve the color constancy problem itself. D'Zmura [4] proposed a method using approximated linear basis functions to form a closed-form equation. One drawback of the method is that it fails to provide robust estimations for real images. Finlayson et al. [8] introduced a method that uses a single surface color illuminated by two different illumination colors. Barnard et al. [2] utilized the retinex algorithm [14] to automatically obtain a surface color with different illumination colors, and then applied the method of Finlayson et al. [8] to estimate varying illumination colors in a scene.

In this paper, our goal is to estimate and to remove the illumination color of outdoor scenes by using a single image. To accomplish this goal, we utilize shadowed and non-shadowed regions. Previously researchers (for example, [7]) have discovered that shadowed regions are illuminated by sky light, while non-shadowed regions are illuminated by a combination of sky light and sunlight. Note that sunlight is due to the direct rays from the sun, and sky light is due to the scattered light rays from the atmosphere. Based on this difference of illumination in shadowed and non-shadowed regions, we have developed a method to estimate the illumination colors (both the sunlight and the sky light) and then remove them. To reliably estimate the illumination colors in outdoor scenes, we include the analysis of noise, since the presence of noise is inevitable in natural images, due to the sensors, the medium, or noise inherent in the objects, such as dust and imperfect painting.

Our basic idea of using shadowed and non-shadowed regions is similar to the idea of using varying illumination $[8,2]$, and our method is principally based on a method proposed by Finlayson et al. [8]. However, unlike the method of Finlayson et al., we take into account the presence of noise, which is inevitable in real images. Finlayson et al. did not include noise in their analyses, which makes their method unreliable for natural images, particularly outdoor scenes. Moreover, instead of using a discrete illumination model, we employ a continuous model that is computed 


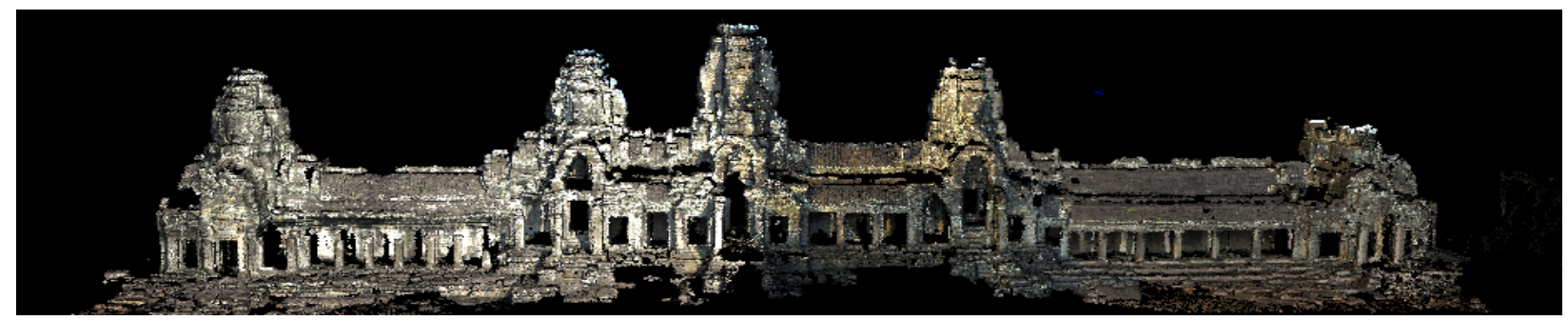

Figure 1. The texture of the Bayon Temple in Angkor, Cambodia. The color of the objects varies due to the use of textures taken at different times.

from the Planck Formula.

To estimate the actual color of the surface successfully, we made the following assumptions: (1) The illumination chromaticity forms a straight line in a two-dimensional inverse-chromaticity space. (2) The camera sensitivity function is narrowband and known. (3) The output of camera response is linear to the flux of incoming light intensity. The last two assumptions are common assumptions used in many color constancy algorithms.

The rest of the paper is organized as follows: in Section 2 , we describe image color formation and the definition of chromaticity. In Section 3, we discuss constraints used in our method. In Section 4, we introduce our approach to make the estimation more robust and accurate. We provide the implementation of our approach and experimental results for real images in Section 5. Finally, in Section 6, we conclude our paper.

\section{Reflection Model}

Image Formation According to general image formation, an image of a diffuse object taken by a digital color camera can be described as:

$$
I_{c}=\int_{\Omega} S(\lambda) E(\lambda) q_{c}(\lambda) d \lambda
$$

where $I_{c}$ is the sensor response (RGB pixel values), $S(\lambda)$ is the surface spectral reflectance and $E(\lambda)$ is the illumination spectral power distribution, $q_{c}$ is the three-element vector of sensor sensitivity, and index $c$ represents the type of sensors $(r, g$ and $b)$. Integration is done over the visible spectrum $(\Omega)$. In this model we ignore camera noise and gain. By assuming narrowband sensitivity that follows the Dirac delta function, Equation (1) can be simply written as:

$$
I_{c}=S_{c} E_{c}
$$

where $S_{c}=S\left(\lambda_{c}\right)$ and $E_{c}=E\left(\lambda_{c}\right)$. If camera sensitivity cannot be approximated by the Dirac delta function (narrowband sensor), we could apply camera sharpening algorithms proposed by $[6,1]$.

Chromaticity Following Finlayson et al. [8], in this paper we define chromaticity (or specifically image chromaticity) as:

$$
\sigma_{c}=\frac{I_{c}}{I_{b}}
$$

where index $c=\{r, g\}$. Equation (2) still holds in this chromaticity space:

$$
\sigma_{c}=s_{c} e_{c}
$$

where $s_{c}$ and $e_{c}$ correspond to the chromaticities of $S_{c}$ and $E_{c}$, which we call surface and illumination chromaticity, respectively.

Planck Formula In this paper, as in many existing color constancy methods, we assume that natural (outdoor) illumination can be approximated by a blackbody radiator, which is modeled by the Planck formula.

The Planck formula is expressed as:

$$
M(\lambda)=c_{1} \lambda^{-5}\left[\exp \left(c_{2} / \lambda T\right)-1\right]^{-1}
$$

where $c_{1}=3.7418 \times 10^{-16} \mathrm{Wm}^{2}, c_{2}=1.4388 \times 10^{-2}$ $\mathrm{mK}, \lambda$ is wavelength $(\mathrm{m})$, and $T$ is temperature in Kelvin. By combining with known sensor sensitivity, we can obtain a camera response of the Planck formula:

$$
I_{c}=\int_{\Omega} M(\lambda, T) q_{c}(\lambda) d \lambda
$$

The last equation is the combination of image formation and the Planck formula.

\section{Estimating Surface Chromaticity}

From Equation (4), the problem of color constancy can be described as the problem of estimating the values of $e_{c}$ and $s_{c}$ given the value of $\sigma_{c}$, where index is $c=\{r, g\}$. However, to estimate four unknown values $\left(e_{r}, e_{g}, s_{r}, s_{g}\right)$ from two known values $\left(\sigma_{r}, \sigma_{g}\right)$ is mathematically illposed. To solve the problem, we should add more constraints, which we do in this paper by increasing the number of the image chromaticities: $\sigma_{c}^{1}$ and $\sigma_{c}^{2}$ that are taken from the same surface chromaticity $\left(s_{c}\right)$ but different illumination chromaticities $\left(e_{c}\right)$ :

$$
\begin{aligned}
\sigma_{c}^{1} & =s_{c} e_{c}^{1} \\
\sigma_{c}^{2} & =s_{c} e_{c}^{2}
\end{aligned}
$$

From the last two equations, we can have four knowns $\left(\sigma_{r}^{1}, \sigma_{g}^{1}, \sigma_{r}^{2}, \sigma_{g}^{2}\right)$, and six unknowns $\left(s_{r}, s_{g}, e_{r}^{1}, e_{g}^{1}, e_{r}^{2}, e_{g}^{2}\right)$, which is mathematically still ill-posed.

Fortunately, from Section 2, we know that natural (outdoor) illumination can be approximately modeled by the 
Planck formula, implying that by using the formula we can have the correlation of $e_{r}^{i}$ and $e_{g}^{i}$, namely, $e_{g}^{i}=f\left(e_{r}^{i}\right)$ where $f$ is a function derived from the Planck formula and index $i=\{1,2\}$. As a result, we can have four unknowns: $\left(s_{r}, s_{g}, e_{r}^{1}, e_{r}^{2}\right)$, and thus the problem has the possibility to be well-posed. In the subsequent section, we will discuss the correlation of $e_{g}$ and $e_{r}$, and then explain how we can automatically have two image chromaticities with the same surface chromaticity but different illumination chromaticity (pixels from shadowed and non-shadowed regions).

\subsection{Illumination Constraints and Shadows}

Illumination Constraints Based on Planck formula (Equation (5)), Marchant et al. [19] derived the correlation of $e_{g}$ and $e_{r}$ as follows:

$$
e_{r}=m e_{g}^{A}
$$

where: $A=\left(\frac{1}{\lambda_{r}}-\frac{1}{\lambda_{b}}\right) /\left(\frac{1}{\lambda_{g}}-\frac{1}{\lambda_{b}}\right), m=\frac{\lambda_{g}^{5 A} / \lambda_{b}^{5 A}}{\lambda_{r}^{5} / \lambda_{b}^{5}}$ and $\left\{e_{r}, e_{g}\right\}$ is the chromaticity of the illumination. $A$ and $m$ are constant numbers characterizing the camera. $\lambda_{c}$ (where index $c=\{r, g, b\})$ is the center wavelength of the camera sensitivity. If we plot this correlation into two-dimensional chromaticity $r g$-space, we can find that all illumination colors form a curved line, which is usually called a Planckian locus.

We have mentioned in the beginning of this section that by knowing the correlation of $e_{g}$ and $e_{r}$, we can probably have a well-posed color constancy problem from two different illuminations, since we have four knowns $\left(\sigma_{r}^{1}, \sigma_{g}^{1}, \sigma_{r}^{2}, \sigma_{g}^{2}\right)$ and four unknowns $\left(s_{r}, s_{g}, e_{r}^{1}, e_{r}^{2}\right)$. Unfortunately, by further derivation from Equation (7), (8) and (9), we obtain the following equations:

$$
\begin{aligned}
& \sigma_{r}^{1}=\frac{s_{r}}{\left(s_{g}\right)^{A}}\left(\sigma_{g}^{1}\right)^{A} \\
& \sigma_{r}^{2}=\frac{s_{r}}{\left(s_{g}\right)^{A}}\left(\sigma_{g}^{2}\right)^{A}
\end{aligned}
$$

The last two equations show that we cannot determine the absolute values of $s_{r}$ and $s_{g}$, since having the same surface chromaticity means that $\left(\sigma_{r}^{1} /\left(\sigma_{g}^{1}\right)^{A}=\sigma_{r}^{2} /\left(\sigma_{g}^{2}\right)^{A}\right)$. Thus, to solve the problem, we should add more constraints, which will be discussed further in Section 3.2.

Two Image Chromaticities with the Same Surface Chromaticity While Equation (10) shows that we cannot have absolute values of $s_{r}$ and $s_{g}$, the equation is useful to determine whether two or more image chromaticities $\left(\sigma_{c}\right)$ have the same surface chromaticity $\left(s_{c}\right)$ but different illumination chromaticity $\left(e_{c}\right)$. The equation shows that $\left(s_{r} /\left(s_{g}\right)^{A}\right)$ can be the same, implying the same $s_{c}$, for different values of image chromaticities $\left(\sigma_{c}\right)$, implying different $e_{c}$. Particularly in outdoor scenes, the equation can be used to detect whether the shadowed and non-shadowed regions are part of the same surface color [19].

\subsection{Straight-Line Constraint}

In the previous section we have shown that solely increasing the number of the inputs $\left(\sigma_{1}\right.$ and $\left.\sigma_{2}\right)$ and having

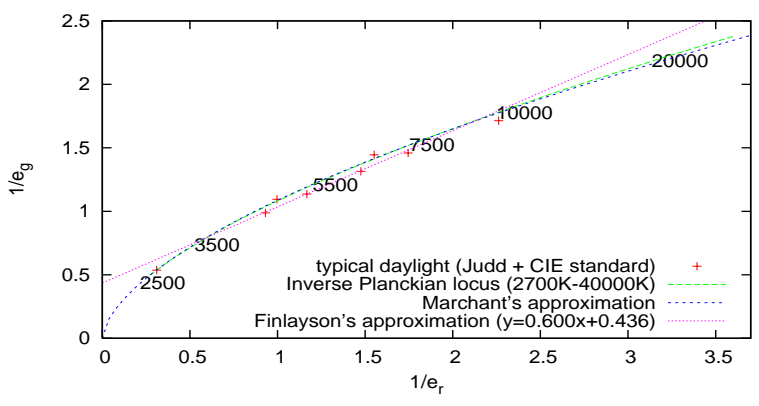

Figure 2. Illumination Models: typical daylight, Planckian locus, Finlayson et al.'s straight-line approximation.

exponential correlation of $e_{g}$ and $e_{r}$ described in Equation (9) are not sufficient to solve the color constancy problem. Following Finlayson et al. [8], we further assume that in two-dimensional inverse-chromaticity $\left(1 / e_{r}, 1 / e_{g}\right)$ space, the illumination can be approximated by a straight line:

$$
\frac{1}{e_{g}}=m \frac{1}{e_{r}}+c
$$

where in Finlayson et al.'s method the values of $m$ and $c$ are constant (not equal to zero), and are computed beforehand. Figure 2 shows a red line that represents the straight line approximation. Based on Equation (12), we have that:

$$
\begin{aligned}
\sigma_{r}^{i} & =s_{r} e_{r}^{i} \\
\sigma_{g}^{i} & =s_{g} \frac{1}{\frac{m}{e_{r}^{i}}+c}
\end{aligned}
$$

where $i=\{1,2\}$. By deriving the last two equations further, we can obtain the following linear correlation:

$$
s_{g}=\left(m \frac{\sigma_{g}^{i}}{\sigma_{r}^{i}}\right) s_{r}+\sigma_{g}^{i} c
$$

The last equation means that in chromaticity space, the image chromaticity $\left(\sigma_{c}\right)$ can form a straight line. This implies that if we have two image chromaticities with the same surface chromaticity $\left(s_{c}\right)$, then their straight lines will intersect at a certain location that is identical to the value of $s_{c}$.

Note that the straight line assumption prevails only for the limited range of $e_{c}^{1}$ and $e_{c}^{2}$. For instance, we cannot use the assumption when the temperature of $e_{c}^{1}$ equals $2500 K$ and the temperature of $e_{c}^{2}$ equals $8000 K$, since, instead of forming a straight line, the illumination chromaticity forms a curved line as shown in Figure 2.

\section{Robust Framework for Outdoor Scenes}

\subsection{Problems}

While Finlayson et al's method elegantly solves the problem of color constancy from varying illumination, we discovered that it has at least two significant drawbacks. The first drawback is that noise is ignored and the second drawback is the assumption that the parameters of the straight line ( $m$ and $c$ in Equation (12)) are constant. 
Noise We have investigated the effects of noise to estimate surface chromaticity in Finlayson et al.'s method quantitatively. Assuming that we have image chromaticity $\sigma_{r}^{1}$ with noise $\Delta \sigma_{r}$, where $\Delta \sigma_{r} / \sigma_{r}^{1} \ll 1$, then the estimated surface chromaticity will deviate from the correct value, described as $s_{c}+\Delta s_{c}$, with $\Delta s_{c}$ representing the error of the estimation. Mathematically, we found that the error ratio of the estimated surface chromaticity can be expressed as by the equation (see Appendix A for detailed derivation):

$$
\frac{\Delta s_{r}}{s_{r}} \approx \frac{\Delta \sigma_{r}}{\sigma_{r}^{1}} \frac{1}{\left(1-\frac{e_{r}^{1} / e_{g}^{1}}{e_{r}^{2} / e_{g}^{2}}\right)}
$$

( As can be seen in the last equation, the error ratio of surface chromaticity $\left(\Delta s_{r} / s_{r}\right)$ will be large if the two illumination colors $e_{c}^{1}$ and $e_{c}^{2}$ are similar. The same analysis can also be done for the green channel. To investigate this further, we simulated the error ratio described in the last equation, and we present the results in Figure 3 . The $y$-axis of the figure represents the error ratio and the $x$-axis represents the temperature of the second illumination in Kelvin. The first simulation is shown in red pointswhen the temperature of the first illumination is $3000 \mathrm{~K}$. As can be observed, when the second illumination's temperature near $3000 K$, the error becomes large. Other simulations using different temperatures of $\sigma_{c}^{1}$ can be observed in the blue and green lines. In this simulation we set $\Delta \sigma_{r} / \sigma_{r}=0.01$.

Thus, if we intend to have relatively accurate results by using Finlayson et al.'s method, the difference between illuminations $\left(e_{c}^{1}\right.$ and $\left.e_{c}^{2}\right)$ should be relatively large. However, a large difference would violate the straight line assumption explained in Section 3.2. We therefore conclude that because of the presence of noise, and because Finlayson et al.'s method is restricted to certain conditions of illumination, the method is unreliable in general conditions of outdoor illumination.

Constant Parameters Moreover, since in Finlayson et al.'s method, $m$ and $c$ in Equation (12) are constants, which are computed from a discrete illumination model proposed by Judd et al's daylight phase and CIE model, this could lead to a wrong estimation for certain conditions of illumination, for example, when the temperature of the first illumination is $2500 \mathrm{~K}$ and the temperature of the second illumination is $4000 \mathrm{~K}$. As shown in Figure 2, the straight line in Finlayson et al.'s method does not pass the inversechromaticity value of illumination whose temperature is $2500 K$.

\subsection{Basic Outline}

In principle, to solve the problems in Finlayson et al.'s method, our ideas are first, to reduce the effect of noise $\Delta \sigma_{c}$ as much as possible so that the final estimation has a relatively small deviation from the ground truth, even if the difference of illumination chromaticity is relatively small; and second, to find the most appropriate values of $m$ and $c$ (the parameters of the illumination straight-line) based on the input chromaticity. The details of the first idea will be discussed in this section, while the second idea will be discussed in Section 4.4.

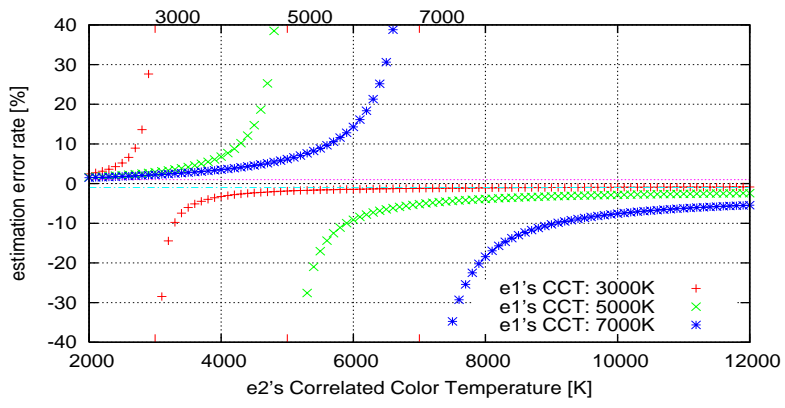

Figure 3. Simulating the effect of noise in estimating the surface chromaticity.

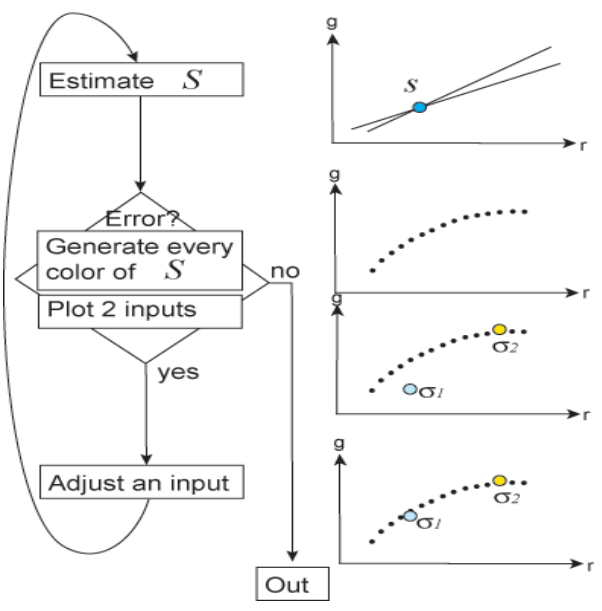

Figure 4. The outline of noise reduction and thus of our robust framework.

Noise Reduction The outline of our noise reduction and thus our robust framework is shown in Figure 4. First, from two image chromaticities $\left(\sigma_{c}^{1}, \sigma_{c}^{2}\right)$, where $\sigma_{c}^{1}$ is taken from a non-shadowed pixel and $\sigma_{c}^{2}$ is taken from a shadowed pixel, we estimate the surface chromaticity $\left(s_{c}\right)$ by solving Equa-
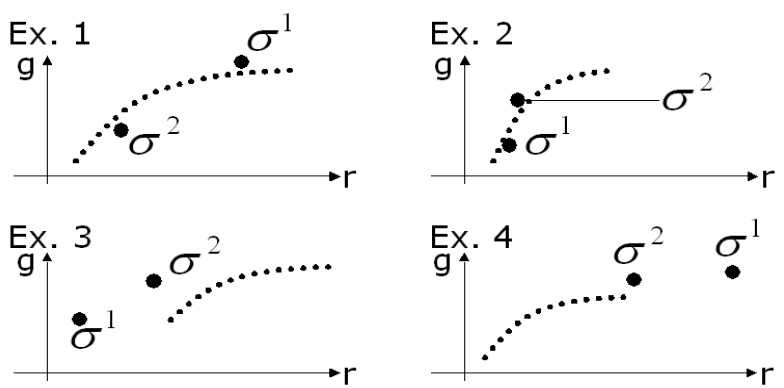

Figure 5. Examples of the distance between the two image chromaticities and the generated possible image chromaticities based on the Planckian locus. 
tion (15), as shown in the top $r g$-space in Figure 4.

Second, we generate all possible image chromaticities based on the estimated surface chromaticity and all possible illuminatian chromaticities, and then plot them into the $r g$-space. The curved dash-line in Figure 4 represents the generated image chromaticities. Mathematically, generating the image chromaticities can be described as:

$$
\sigma_{c}^{\text {planck }}=s_{c} e_{c}^{\text {planck }}
$$

where $e_{c}^{\text {planck }}$ is the chromaticity of Planckian locus, i.e., the colors of all natural illumination, $s_{c}$ is the estimated result, and $\sigma_{c}^{\text {planck }}$ is the generated image chromaticity.

Third, we examine the correctness of our estimated surface chromaticity by the following rule: If the estimated surface chromaticity is correct then the two image chromaticities $\left(\sigma_{c}^{1}, \sigma_{c}^{2}\right)$ must lie on the generated curved line; otherwise, $\sigma_{c}^{1}, \sigma_{c}^{2}$ do not lie on the curved line. Examples of inputs that largely lie on the curved line and that do not lie in the curved line are shown in Figure 5. Ex. 1 and Ex. 2 are examples of inputs that largely lie on the curved line, and Ex. 3 and Ex. 4 are examples of inputs that do not lie on the curved line.

Fourth, if the inputs largely lie on the curved line, then the process terminates and we can have a relatively correct result. Otherwise, we consider that one or two of the input chromaticities have noise. To reduce this noise, we change the position by adjusting the value of the image chromaticity slightly. After the adjustment, we estimate the value of $s_{c}$ once again and then detect whether the inputs largely lie on the curved line. The process is done iteratively until the inputs largely lie on the curved line. In practice we use Euclidean distance to determine whether the inputs lie on the curved line.

\subsection{Image-chromaticity Adjustment}

There are two issues in adjusting the values of image chromaticity that has noise. First, from two image chromaticities $\left(\sigma_{c}^{1}\right.$ and $\sigma_{c}^{2}$ ), we should choose which chromaticity has more noise than the other. This becomes an issue because we intend to adjust one image chromaticity instead of two. We found that if we adjust both image chromaticities, ambiguity will result. Consider Equations (10) and (11) and assume that both $\sigma_{c}^{1}$ and $\sigma_{c}^{1}$ have noise $\left(\Delta \sigma_{c}^{1}\right.$ and $\left.\Delta \sigma_{c}^{2}\right)$, where at first their values are different $\left(\Delta \sigma_{c}^{1} \neq \Delta \sigma_{c}^{2}\right)$, meaning the values of $s_{r} /\left(s_{g}\right)^{A}$ will be different. Then we adjust both of them. This adjustment could lead to a certain condition where the values of $\left(s_{r} /\left(s_{g}\right)^{A}\right)$ are the same, but it does not guarantee that $\Delta \sigma_{c}^{1}=\Delta \sigma_{c}^{2}=0$.

Therefore, we decided to choose only one of the inputs to be modified to reduce the error. This constraint brings us two benefits. First, the processing time becomes fast. Second, we are sure that the processing always terminates. In our implementation, we chose the pixel from a shadowed region to be adjusted, since the darker pixels in general have much noise than brighter pixels.

Second, upon choosing the image chromaticity to be adjusted, we have to decide in which direction the adjustment has to be done. This issue is due to the random value of noise, which could be positive and negative. To solve the problem, first we adjust the input in either vertical direction (green-channel) or horizontal direction (red-channel), which we determine by using following equation (see Appendix $\mathrm{B}$ for the detailed derivation):

$$
\frac{\left(\sigma_{r}^{B}-\sigma_{r}^{A}\right)}{\left(\sigma_{g}^{B}-\sigma_{g}^{A}\right)} \frac{\sigma_{r}^{A} / \sigma_{r}^{B}}{\sigma_{g}^{A} / \sigma_{g}^{B}}>1
$$

If the left side of the last equation is larger than 1 , then $\Delta \sigma_{g} / \sigma_{g}$ has a greater effect on the estimation. Thus, we move the image chromaticity in parallel with the green channel (vertical direction). Second, to determine whether the adjustment should be upward or downward (in the case of vertical direction, or right or left in the case of horizontal direction), we use the iteration procedure we have explained in Section 4.2, namely, at first we apply positive adjustment. If the distance between the adjusted image chromaticity and the generated curved line is larger than that without adjustment then we should apply negative adjustment, or viceversa.

\subsection{Temperature Range}

As we have mentioned, one of the main drawbacks of Finlayson et al.'s method is that they set the values of $m$ and $c$ constant, which could lead to violating the straight line assumption. We solve this problem by having more than single values for $m$ and $c$. The value depends on the range of illumination. For outdoor illumination, normally we can have from $2500 \mathrm{~K}$ to $10000 \mathrm{~K}$. Thus, we divide the range into two ranges: $2500 \mathrm{~K}-4000 \mathrm{~K}$ and $4000 \mathrm{~K}-10000 \mathrm{~K}$, producing two pairs of $m$ and $c:\left\{m_{1}, c_{1}\right\}$ and $\left\{m_{2}, c_{2}\right\}$. For indoor illumination, the range and thus the number of pairs could be different.

In the computation, to choose the correct $m$ and $c$, we use a method similar to that for noise reduction: more correct $m$ and $c$ will make the generated image chromaticity $\left(\sigma_{c}^{\text {planck }}\right)$ closer to the input chromaticity $\left(\sigma_{c}^{1}\right.$ and $\left.\sigma_{c}^{2}\right)$.

\section{Implementation and Experimental Results}

\subsection{Implementation}

The implementation of our method is as follows. First, from an input image that has shadows (as an example see Figure 6.a), we compute its image chromaticity by using Equation (3). Then, we find pixels from a shadowed region and a non-shadowed region that have the same surface chromaticity or the same value of $\left(s_{r} /\left(s_{g}\right)^{A}\right)$. Figure 6.b shows an image representing the values of $\left(s_{r} /\left(s_{g}\right)^{A}\right)$. If we plot this value in chromaticity space, then we can obtain clustered points, as shown in Figure 7.a. The blue line represents the same value of $\left(s_{r} /\left(s_{g}\right)^{A}\right)$, implying the same value of surface chromaticity.

Upon knowing the values of $\left(s_{r} /\left(s_{g}\right)^{A}\right)$, we can obtain two or more pixels that have the same value of $\left(s_{r} /\left(s_{g}\right)^{A}\right)$ but different values of image chromaticity $\left(\sigma_{c}\right)$, namely, pixels from shadowed and non-shadowed regions, by analyzing the points that lie on the blue line shown in Figure 7.a. If we compute the histogram of points lying on the blue line, we will obtain a distribution shown in Figure 7.b (the red lines). As shown in the figure, we have two peaks. The 


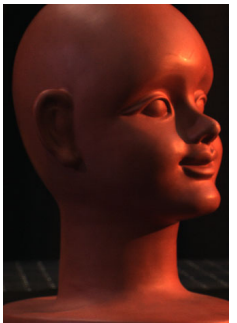

(a)

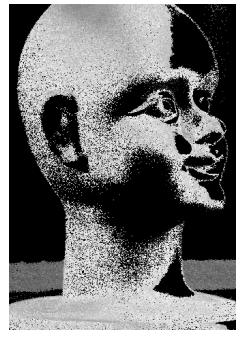

(b)

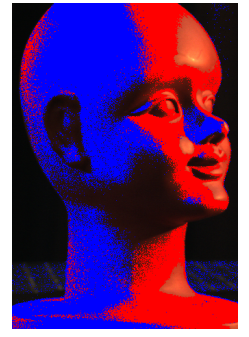

(c)
Figure 6. Result of indoor experiment, by using artificial lights.

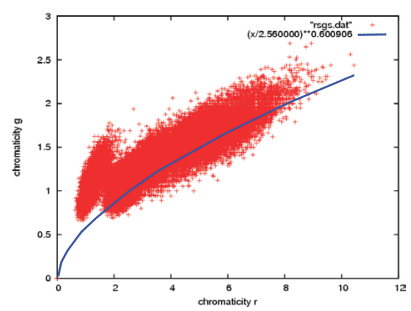

(a)

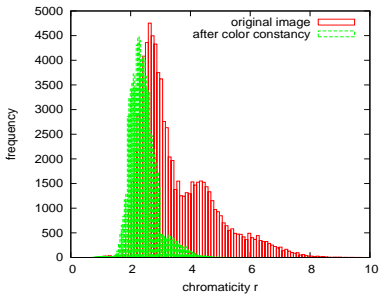

(b)
Figure 7. (a) Points that have the same value of $\left(s_{r} / s_{g}^{A}\right)$ plotted in rg-space. (b) Comparison results between the histogram of input image chromaticities and that of output image chromaticities in the red channel

left peak represents the first illumination chromaticity (nonshadowed region), and the right peak represents the second illumination chromaticity (shadowed region). By finding the two peaks, we can have pixels representing different illumination chromaticity but the same surface chromaticity. From those pixels, we can use the algorithm explained in Section 4 to estimate the illumination chromaticities.

Then, having estimated the illumination chromaticities, we intend to obtain the surface chromaticities of all pixels in the input image. To do this we use a simple approach. From Figure 7.b, we can have two peaks representing the shadowed and non-shadowed regions. If we cluster the pixels according to the peaks, we can have all pixels representing the shadowed region and all pixels representing the non-shadowed region. The result can be seen in Figure 6.c, where blue pixels represent the shadowed region and red pixels represent the non-shadowed region. Then, we can compute the surface chromaticity simply by dividing the image chromaticity by the estimated illumination chromaticity. The result of removing one of the illumination colors can be seen in the distribution of the green lines in 7.b. Unlike the red lines, the green lines only have one peak.

\subsection{Experimental Result}

Conditions We conducted several experiments on real images, taken using SONY DXC-9000 and Nikon-D1 progressive 3 CCD digital cameras, by setting their gamma cor-
Table 1. Comparison of estimated illumination chromaticities resulting from our method and from Finlayson et al.'s method.

\begin{tabular}{l|ll}
\hline & $\begin{array}{l}\text { Average } \\
\text { Error }\end{array}$ & $\begin{array}{l}\text { Maximum } \\
\text { Error }\end{array}$ \\
\hline Our Estimation & 0.063 & 0.16 \\
Finlayson et al.'s Estimation & 0.11 & 0.32 \\
\hline
\end{tabular}

rection off. To ensure that the outputs of the cameras were linear to the flux of incident light, we used the Macbeth color chart. We used planar and convex objects to avoid inter-reflection, and excluded saturated pixels and pixels below the camera dark from the computation. For evaluation, we compared the results with the average values of image chromaticity of a white reference image (Photo Research Reflectance Standard model SRS-3), captured by the same cameras.

Evaluation We have conducted a number of experiments using the Macbeth color chart in outdoor illumination. One of the experiments was done by using a green surface taken under cloudy daylight conditions at 16:30 and 17:30. The illumination chromaticity taken from the white reference were $(0.403,0.310)$ and $(0.456,0.305)$. Using Finlayson et al.'s method, the estimations were $(0.525,0.288)$ and $(0.533,0.285)$, while using our method, the estimations were $(0.401,0.324)$ and $(0.409,0.322)$. We also calculated the average error and the maximum error of our method compared with Finlayson et al.'s method, as shown in Table 1 . The total number of images in our experiments was 30. As shown in Table, our method produced more accurate and robust results. The error in the table was computed based on chromaticity defined by standard CIE.

Outdoor Scenes The input of our experiment is shown in Figure 8.a, and the image chromaticity of the input is shown in Figure 8.b. Figure 8.c shows the values of $\left(s_{r} /\left(s_{g}\right)^{A}\right)$. The same values of $\left(s_{r} /\left(s_{g}\right)^{A}\right)$ represent the same surface chromaticity. From the input chromaticity (Figure 8.b.) and Figure 8.c, we determine pixels that correspond to shadowed regions and non-shadowed regions of the same surface chromaticity. Figure 8.d shows the shadowed region (blue pixels) and non-shadowed region (red pixels). Note that, in this paper, we do not intend to detect or segment the shadowed region; the red and blue pixels only represent two image chromaticities we used for color constancy (thus we do not need to precisely cluster the pixels). Figure 8.e shows the result of color constancy, and Figure 8.f shows its image chromaticity. Notice that compared with Figure 8.b which has different image chromaticity in the shadowed area, Figure 8.f shows that there is no longer any difference of illumination color.

Besides using shadows from a single image to evaluate the robustness of our method, we also conducted experiments by using two images taken at different times, and thus having different colors of illumination. Figure 9.a and 9.b show the input images. By using 3D geometrical data (provided by a laser range sensor), we obtained the corresponding location of each surface point. For the two different pixels taken from the same point, we estimated the illumination and then removed the illumination color. Figure 9.c shows our estimated surface color, while Figure 9.d shows 
the result by using the white reference.

\section{Conclusion}

We have proposed a method to estimate surface color from shadows. Our main contribution is to develop a method that is robust and accurate even for outdoor objects, where conditions are less controllable compared with conditions for indoor objects. The underlying idea of our approach is to reduce noise and to find the most appropriate parameters of the straight-line assumption. The experimental results on outdoor scene show the effectiveness of our method.

\section{Acknowledgments}

This work was, in part, supported by Ministry of Education, Culture, Sports, Science and Technology, under the program, "Development of High Fidelity Digitization Software for Large-Scale and Intangible Cultural Assets".

\section{Appendix A}

The surface chromaticity $s_{r}$ can be derived as:

$$
s_{r}=\frac{\sigma_{r}^{1} \sigma_{r}^{2}\left(\sigma_{g}^{2}-\sigma_{g}^{1}\right)}{\sigma_{r}^{2} \sigma_{g}^{1}-\sigma_{r}^{1} \sigma_{g}^{2}} \frac{c}{m}
$$

If input $\sigma_{r}^{1}$ has noise $\Delta \sigma_{r}^{1}$, then the estimated surface chromaticity becomes $s_{r}+\Delta s_{r r}$ :

$$
s_{r}+\Delta s_{r r}=\frac{\left(\sigma_{r}^{1}+\Delta \sigma_{r}^{1}\right) \sigma_{r}^{2}\left(\sigma_{g}^{2}-\sigma_{g}^{1}\right)}{\sigma_{r}^{2} \sigma_{g}^{1}-\left(\sigma_{r}^{1}+\Delta \sigma_{r}^{1}\right) \sigma_{g}^{2}} \frac{c}{m}
$$

Thus, the error ratio $\Delta s_{r r} / s_{r}$ can be calculated as:

$$
\frac{\Delta s_{r r}}{s_{r}}=\frac{\Delta \sigma_{r}^{1}}{\sigma_{r}^{1}} \frac{1}{1-\frac{\sigma_{r}^{1} / \sigma_{g}^{1}}{\sigma_{r}^{2} / \sigma_{g}^{2}}-\frac{\Delta \sigma_{r}^{1}}{\sigma_{r}^{1}} \frac{\sigma_{r}^{1} / \sigma_{g}^{1}}{\sigma_{r}^{2} / \sigma_{g}^{2}}}
$$

Since we assume $\Delta \sigma_{r}^{1} / \sigma_{r}^{1} \ll 1$, the equation becomes:

$$
\frac{\Delta s_{r r}}{s_{r}} \approx \frac{\Delta \sigma_{r}^{1}}{\sigma_{r}^{1}} \frac{1}{1-\frac{\sigma_{r}^{1} / \sigma_{g}^{1}}{\sigma_{r}^{2} / \sigma_{g}^{2}}}
$$

\section{Appendix B}

Using the same derivation in Appendix A, when $\sigma_{g}^{1}$ has noise $\Delta \sigma_{g}^{1}$, then the error ratio of estimated surface chromaticity becomes:

$$
\begin{aligned}
\frac{\Delta s_{r g}}{s_{r}} & \approx-\frac{\Delta \sigma_{g}^{1}}{\sigma_{g}^{1}} \frac{1}{1-\frac{\sigma_{r}^{1} / \sigma_{g}^{1}}{\sigma_{r}^{2} / \sigma_{g}^{2}}} \frac{\left(\sigma_{r}^{2}-\sigma_{r}^{1}\right) \sigma_{r}^{1} / \sigma_{r}^{2}}{\left(\sigma_{g}^{2}-\sigma_{g}^{1}\right) \sigma_{g}^{1} / \sigma_{g}^{2}} \\
& \approx \frac{\Delta s_{r r}}{s_{r}} \frac{\left(\sigma_{r}^{2}-\sigma_{r}^{1}\right) \sigma_{r}^{1} / \sigma_{r}^{2}}{\left(\sigma_{g}^{2}-\sigma_{g}^{1}\right) \sigma_{g}^{1} / \sigma_{g}^{2}}
\end{aligned}
$$

This shows that even the error ratio of $\sigma_{r}^{1}$ and $\sigma_{g}^{1}$ are the same, the effect on the estimation error ratio depends on the factor $\frac{\left(\sigma_{r}^{2}-\sigma_{r}^{1}\right) \sigma_{r}^{1} / \sigma_{r}^{2}}{\left(\sigma_{g}^{2}-\sigma_{g}^{1}\right) \sigma_{g}^{1} / \sigma_{g}^{2}}$.

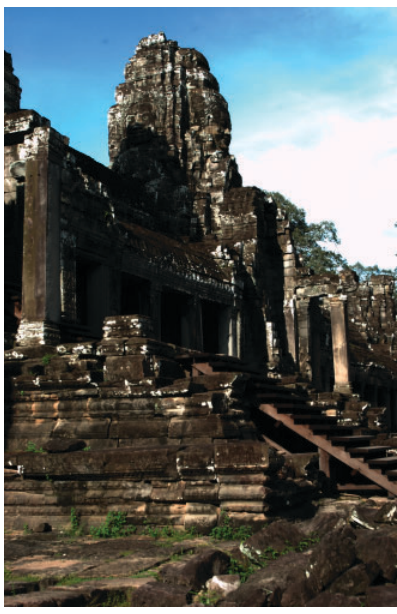

(a)

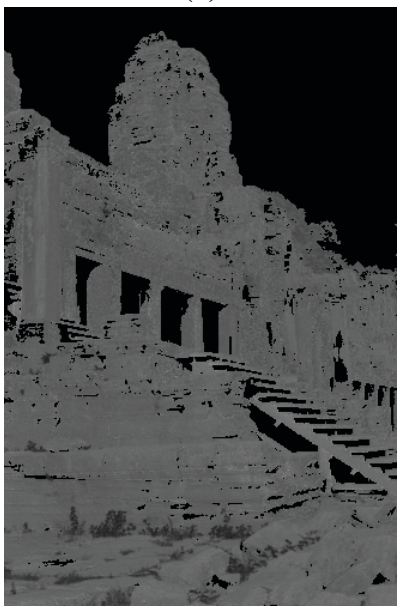

(c)

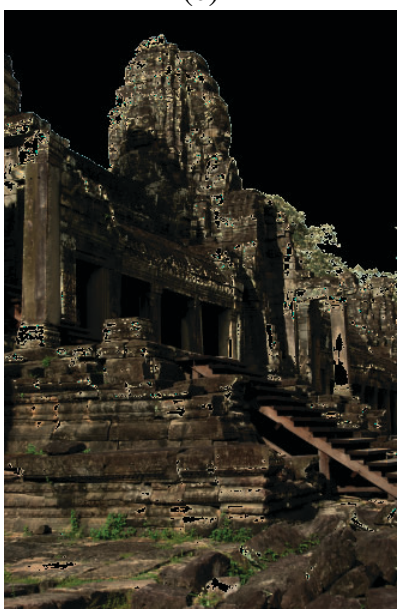

(e)

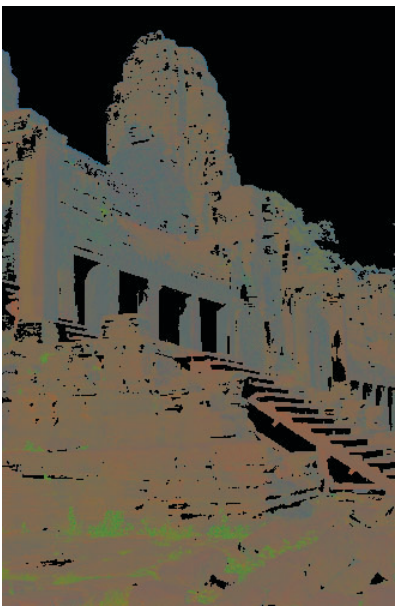

(b)

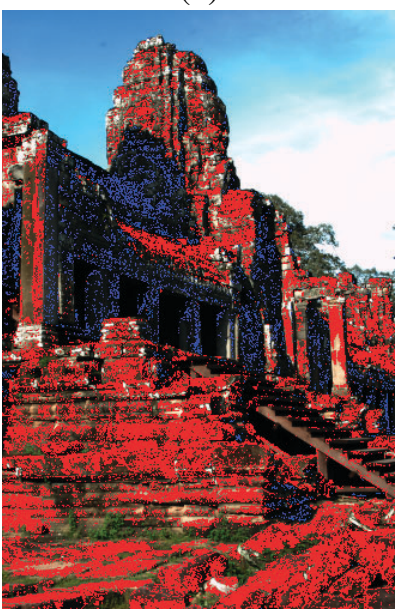

(d)

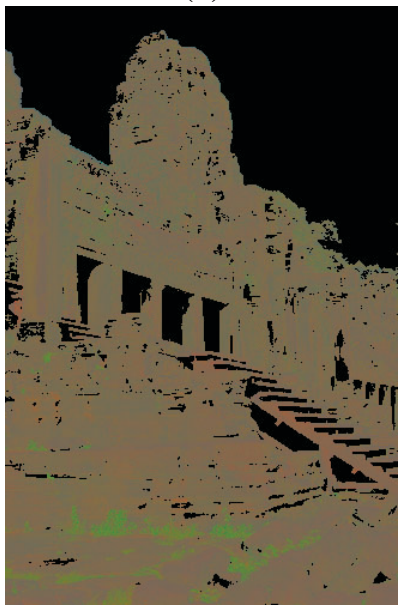

(f)
Figure 8. Results of outdoor experiment: (a) input image (b) input chromaticity (c) the image of the values of $\left(s_{r} /\left(s_{g}\right)^{A}\right)(d)$ the shadowed (blue) and nonshadowed (red) pixels of the same surface chromaticity $(e)$ the result of color constancy $(f)$ the result in chromaticity. 


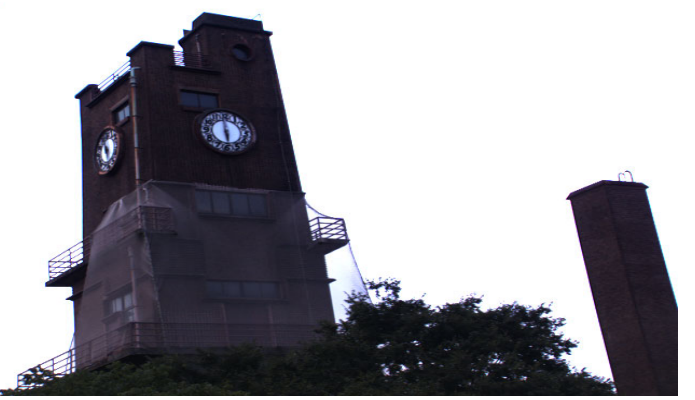

(a)

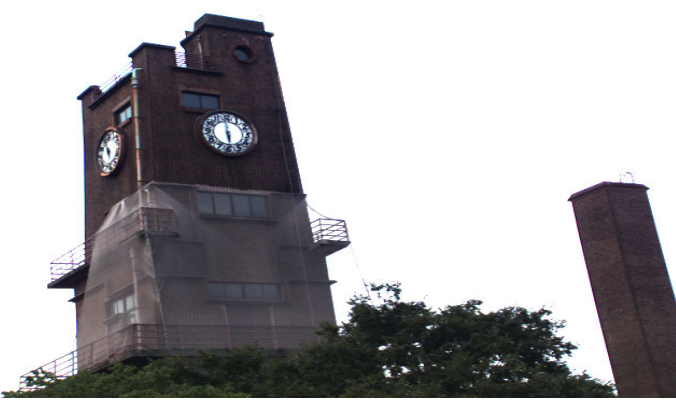

(c)

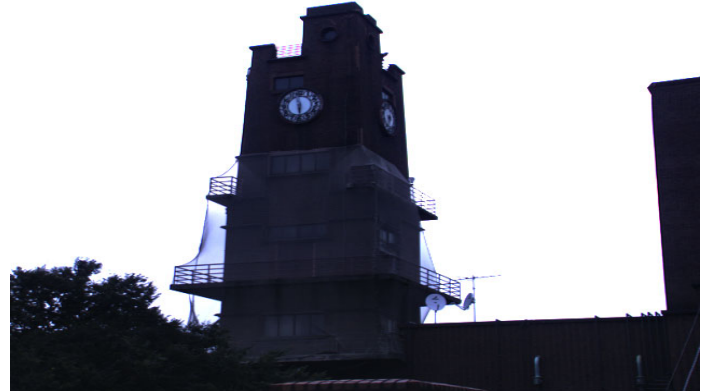

(b)

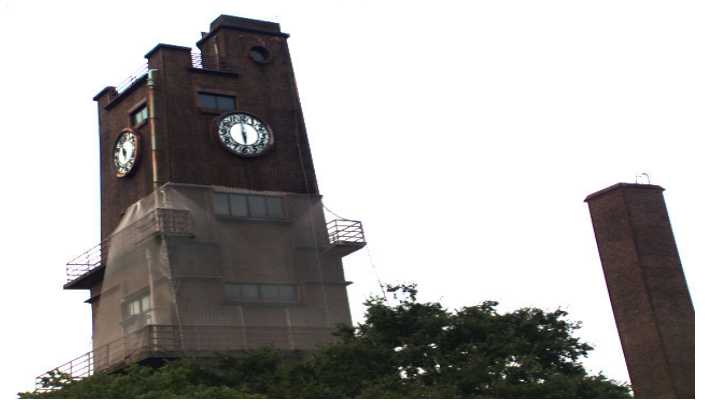

(d)

Figure 9. (a) One of the two input scenes, illuminated by cloudy daylight at 18:00. (b) The other input, illuminated by cloudy daylight at 18:00 on another day. (c) The estimated scene's actual color of the image shown in (a) computed using our proposed method. (d) The estimated scene's actual color using the standard white reference.

\section{References}

[1] K. Barnard, F. Ciurea, and B. Funt. Sensor sharpening for computational color constancy. Journal of Optics Society of America A., 18(11):2728-2743, 2001.

[2] K. Barnard, G. Finlayson, and B. Funt. Color constancy for scenes with varying illumination. Computer Vision and Image Understanding, 65(2):311-321, 1997.

[3] D.H. Brainard and W.T. Freeman. Bayesian color constancy. Journal of Optics Society of America A., 14(7):1393-1411, 1997.

[4] M. D'Zmura. Color constancy: surface color from changing illumination. Journal of Optics Society of America A., 9(3):490-493, 1992.

[5] M. D'Zmura and P. Lennie. Mechanism of color constancy. Journal of Optics Society of America A., 3(10):1162-1672, 1986.

[6] G.D. Finlayson, M.S. Drew, and B.V. Funt. Spectral sharpening sensor transformations for improved color constancy. Journal of Optics Society of America A., 11(10):1162-1672, 1994.

[7] G.D. Finlayson and B.V. Funt. Color constancy using shadows. Perception, 23:89-90, 1994.

[8] G.D. Finlayson, B.V. Funt, and K. Barnard. Color constancy under varying illumination. in proceeding of IEEE International Conference on Computer Vision, pages 720-725, 1995.

[9] G.D. Finlayson, S.D. Hordley, and P.M. Hubel. Color by correlation: a simple, unifying, framework for color constancy. IEEE Trans. on Pattern Analysis and Machine Intelligence, 23(11):1209-1221, 2001.
[10] G.D. Finlayson and S.D.Hordley. Color constancy at a pixel. Journal of Optics Society of America A., 18(2):253-264, 2001.

[11] B.V. Funt, M. Drew, and J. Ho. Color constancy from mutual reflection. International Journal of Computer Vision, 6(1):524, 1991.

[12] J.M. Geusebroek, R. Boomgaard, S. Smeulders, and T. Gevers. A physical basis for color constancy. In The First European Conference on Colour in Graphics, Image and Vision, pages 3-6, 2002.

[13] D.B. Judd, D.L. MacAdam, and G. Wyszecky. Spectral distribution of typical daylight as a function of correlated color temperature. Journal of Optics Society of America, 54(8):1031-1040, 1964.

[14] E.H. Land and J.J. McCann. Lightness and retinex theory. Journal of Optics Society of America, 61(1):1-11, 1971.

[15] H.C. Lee. Method for computing the scene-illuminant from specular highlights. Journal of Optics Society of America A., 3(10):1694-1699, 1986.

[16] H.C. Lee. Illuminant color from shading. In Perceiving, Measuring and Using Color, page 1250, 1990.

[17] R. T. Tan, K. Nishino, and K. Ikeuchi. Color constancy through inverse intensity-chromaticity space. Journal of the Optical Society of America A (JOSA A), 21(3):321-334, 2004.

[18] S. Tominaga and B.A. Wandell. Natural scene-illuminant estimation using the sensor correlation. Proceedings of the IEEE, 90(1):42-56, 2002.

[19] J.A. Marchant and C.M. Onyango. Shadow-invariant classification for scenes illuminated by daylight. Journal of Optics Society of America A. 17(11):1952-1961, 2000. 